\title{
SENTINEL FOR APPLICATIONS IN AGRICULTURE
}

\author{
Mario A. Gomarasca ${ }^{1, *}$, Antonella Tornato ${ }^{2}$, Daniele Spizzichino ${ }^{2}$, Emiliana Valentini ${ }^{2}$, Andrea Taramelli ${ }^{2,}$, Giuseppe Satalino ${ }^{3}$, \\ Massimo Vincini ${ }^{4}$, Mirco Boschetti ${ }^{5}$, Roberto Colombo ${ }^{6}$, Livio Rossi ${ }^{7}$, Enrico Borgogno Mondino ${ }^{8}$, Luigi Perotti ${ }^{9}$, Walter Alberto ${ }^{9}$, \\ Fabio Villa ${ }^{9}$ \\ 1,* Summer School Director, Italian Remote Sensing Association (AIT) \& CNR-IREA Milano, Italy-gomarasca.m@irea.cnr.it \\ ${ }^{2}$ ISPRA, Rome, Italy, ${ }^{\S}$ IUSS, Pavia, Italy, ${ }^{3} \mathrm{CNR}$ IREA Bari, Italy, ${ }^{4} \mathrm{CR} A S T$-UNIPC, Piacenza, Italy, ${ }^{5} \mathrm{CNR}$ IREA Milano, Italy, \\ ${ }^{6}$ DISAT-UNIMIB, Milano, Italy, e-GEOS SpA, Rome, Italy, ${ }^{8}$ DISAFA-UNITO, Torino, Italy, ${ }^{7}$ IMAGEO srl Spin-off, Torino, Italy
}

\author{
Commission III, WG III/10
}

KEY WORDS: Copernicus, Sentinel, Agriculture, Earth Observation, Geo-information, Geomatics

\begin{abstract}
:
The European Union and the European Space Agency (EU/ESA) have promoted since 1998 (Baveno Manifesto*) the GMES Programme (Global Monitoring for Environment and Security), nowadays called Copernicus (www.copernicus.eu). In the agriculture domain, the use of Copernicus Sentinel imagery and its services are providing several new opportunities. The knowledge of fundamentals of Earth Observation/Geographic Information EO/GI, namely Geomatics, for the development of innovative strategies for professional skills adequacy and capacity building, supporting Copernicus user uptake, becomes mandatory (Gomarasca, 2009). The target is to help bridging gaps between supply and demand of education and training for geospatial sector (www.eo4geo.eu). The innovative and strategical novelties are the complete free access to Sentinel time series imagery and digital image processing software "Sentinel toolboxes" such as SNAP (Sentinel Application Platform) for different environments (Windows, Mac, Unix). The paper introduce topics as crop mapping and monitoring, biophysical parameters, phenology and yield estimations, through several concluded or ongoing international projects such as: ERMES -FP7 (http://www.ermes-fp7space.eu/it/homepage/, Busetto et al. 2017) and SATURNO (https://www.progettosaturno.it/, Nutini et al., 2018) devoted to the regional agricultural monitoring. As conclusion, SNAP software for image processing of Sentinel data was demonstrated and tested together with Earth Engine software for specific vertical agriculture applications. The topics reported in this paper have been part of the Summer School 'Sentinel for Applications in Agriculture' supported by the Copernicus programme, several scientific associations (AIT, ASITA, EARSeL - European Association of Remote Sensing Laboratories), the European Erasmus+ project EO4GEO, University Departments and Geo-Information Companies.
\end{abstract}

\section{INTRODUCTION}

The Copernicus Programme, the EU Earth Observation and monitoring programme, was established by Regulation (EU) No $377 / 2014$ of the European Parliament and of the Council, on April $3^{\text {rd }}, 2014$ (http://www.copernicus.eu/).

The programme was designed to provide a European response to Global needs such as the environment management, climate change effects mitigation and to ensure civil and citizen security. More in detail the programme is a cornerstone of the European Union's efforts to monitor the Earth and it's many ecosystem, whilst ensuring that its citizens are prepared and protected in the faces of crises and natural and man-made disasters. Copernicus is also a useful tool for economic development and a driver for digital economy. The programme entered its operational phase with the launch of Sentinel-1A in 2014 and its governance is based on the Copernicus Regulation adopted the same year witch established the Commission as the
Programme manager owing the infrastructure and data rights on behalf of the Union. The Space segment and services are based on information from a dedicated constellation of satellites (Sentinels), as well as of the third-party satellites known as "contributing space missions", complemented by "in situ" (meaning local or on site) measurement data and the added value products. By making the vast majority of its data, analysis, forecast and maps available and accessible, Copernicus contribute towards the development of new innovative applications and services, tailored to the need of specific groups of user, which touch on a variety of economic and cultural or recreational activities, from Urban Planning, sailing and insurance to archeology. From the economic point of view the programme is supported by an adequate and substantial budget. The Overall programme is coordinate by European Commission while the space component is coordinated by ESA and public and private partners. Future development concerning the space component will be a joint EC-ESA divided into two different sets of activities: Sentinel 
future Expansion and Next Generation Sentinels. The other fundamental pillar of the programme are the six cross cut thematic services: Land monitoring, marine environment, Atmosphere, Climate Change, Emergency Management and Security. The Copernicus Programme is designed and operates to make available to several public and private user communities trusted and quality multi-source products and services. The engagement of Member States takes place in the frame of the National User Forum, and is characterized by some common denominators: coping with the European and national obligations, facilitating and sustaining the scientific and technologic innovation to realize downstream services and societal benefits through the National Space Economy and Policy. Finally, since the development of operational services has to be user-driven oriented, the criteria for Sentinel evolution are:

- consolidated user needs: Copernicus Core users require additional observational capacity,

- gap analysis: new mission taking into account synergies with other missions and the pool of current/novel products.

- technological readiness: the mission could be realized within the timeframe 2025-2030. Detailed criteria for application/technology/in-situ.

- affordability: mission can be realized with realistic assumptions on available budgets to meet requirements effectively or efficiently.

\section{Description of the activities and discussion}

\subsection{Sentinel-1 for agriculture}

The potentiality of Sentinel-1 for crop mapping comes from the sensitivity of Synthetic Aperture Radar (SAR) data to crop structure and vegetation water content. Although optical sensors (e.g. Sentinel-2) are well suited for agricultural applications, the use of Sentinel-1 can offer an additional and complementary source of information for crop mapping, as Sentinel-1 SAR images: i) are not affected by cloud cover, ii) have a global coverage, and iii) have a frequent temporal revisit. The Sentinel-1 constellation has been designed to acquire high resolution dual-polarized C-band SAR data with a temporal gap of 6 days. This is achieved by using two satellites (i.e. S-1A and S-1B) sharing the same orbit plane with a $180^{\circ}$ orbital phasing difference. The C-SAR instrument on-board can operate in four exclusive acquisition modes (i.e. i) Strip map (SM), ii) Interferometric Wide swath (IW), iii) Extra-Wide swath (EW), and iv) Wave mode (WV)), with different resolution (down to $5 \mathrm{~m}$ ) and coverage (up to $400 \mathrm{~km}$ ). The obtained acquisitions are made available as SAR products with different level of processing and resolution (e.g. level-1 SLC, level-1

GRD)

(https://sentinel.esa.int/web/sentinel/missions/sentinel-

1/overview). SAR products can be searched and downloaded by means of the Copernicus Open Access Hub (https://scihub.copernicus.eu/). Among them, the most used product for agricultural applications is the IW Level-1 GRDH product. This product needs to be pre-processed before its use, following a typical chain of pre-processing that includes: calibration, co-registration, multi-looking and geocoding. This pre-processing can be performed by using the Sentinel-1 Toolbox (S1TBX), which consists of a collection of processing tools, data product readers and writers, embedded into the SNAP common architecture (https://sentinel.esa.int/web/sentinel/toolboxes/sentinel-1). The crop classification by using Sentinel-1 data is a specific problem of data classification. A wide literature is available concerning classification methods in general (e.g. supervised or unsupervised) and crop classification methods from SAR data in particular (Oliver and Quegan, 1998). What have to be taken into account is that crop classification accuracy depends not only on the specific method used but also on various factors such as:

- level of data pre-processing (speckle filtering, multitemporal filtering, ...);

- SAR features used (backscatter coefficients, singledual polarization, multi-temporal data, ...);

- type of crops (winter-summer crops, small stem crops, broad leaf plants, ...);

- number of crops.

A comprehensive overview of the results achievable by means of supervised approaches as applied to multi-polarized and/or multi-temporal SAR data can be found in Skriver et al., 2011, where the importance of the polarizations (e.g. VH and VV) and of the use of multi-temporal SAR data for improving the crop classification accuracy are illustrated. Their main drawback is that the classification methodology is data driven (i.e., training fields sampled on the investigated site are required); therefore, the classifier performance is normally space and time dependent. As an example, a winter crop map obtained from multi-temporal Sentinel-1 images acquired in 2015 over the Apulian Tavoliere (Southern Italy) is given in Figure 1. The map identifies seven soil/crop classes and it has been obtained by a classification procedure of seven multitemporal VV and VH Sentinel-1 pre-processed images (at approximately $100 \mathrm{~m}$ pixel resolution) acquired during spring 2015 and by using the Maximum Likelihood classifier. The overall accuracy of the map, evaluated over a testing data set, achieves $85 \%$. As can be observed, the majority of the agricultural area is covered by cereals, i.e. wheat, barley and oat crops.

Sentinel-1 data have a good potential to identify agricultural crops due to its sensitivity to crop structure and vegetation water content. This potential can be exploited to get crop maps with a good classification accuracy (e.g. 85\%) by using multitemporal and dual polarization (VV and VH) Sentinel-1 data.

\subsection{Sentinel-2 for agriculture}

Sentinel-2 constellation is built to analytically acquire highresolution, multi-spectral images with a high revisit time globally. The constellation is composed by two (Sentinel-A and B) polar orbiting satellites space out at $180^{\circ}$ visible in Figure 2. The Multi-Spectral Instrument (MSI) is the sensor carried as 


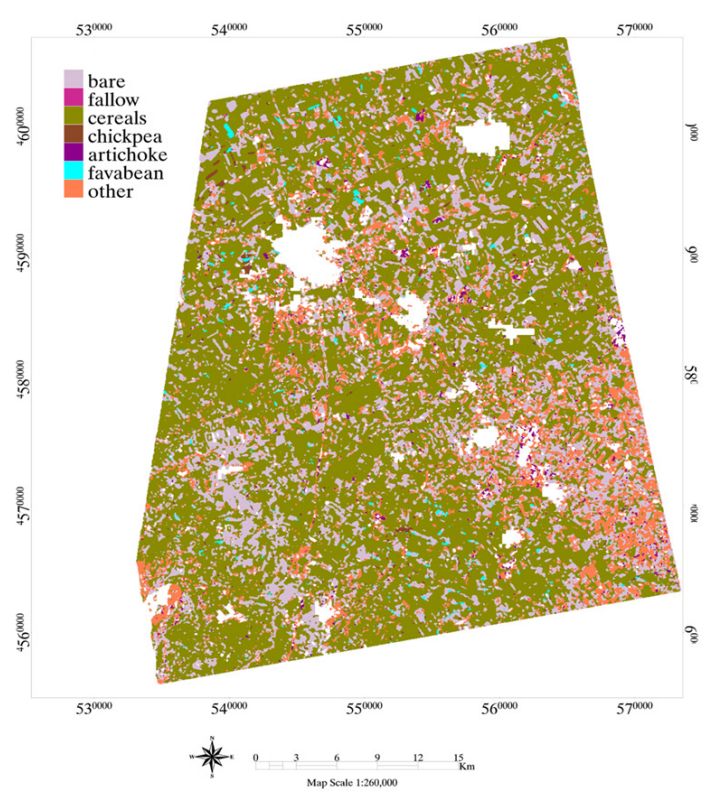

Figure 1. Winter crop map over the Apulian Tavoliere site (Italy) derived from multi-temporal Sentinel-1 images in 2015.

payload and has a push-broom configuration. The MSI sensor acquires in 13 different spectral band (Visible, NIR and SWIR) with a ground sample resolution (GSD) of $10 \mathrm{~m}$ for (B2, B3, B4 and B8) and a swat of $290 \mathrm{~km}$.
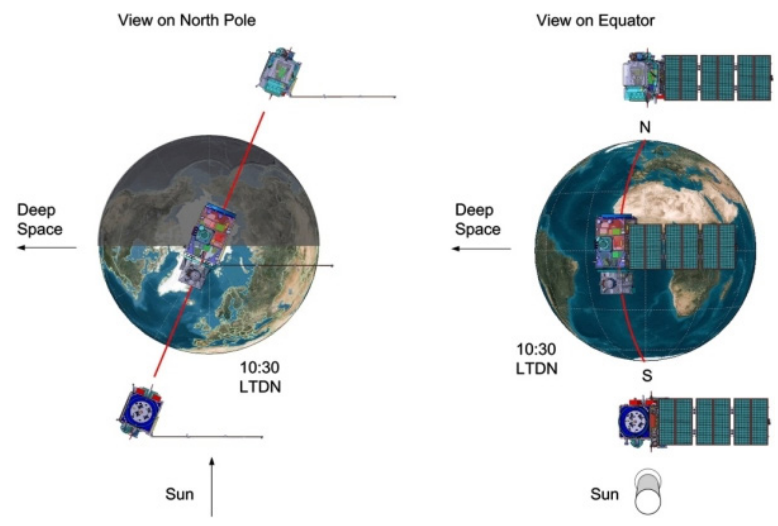

Figure 2. Sentinel-2 overview (source:

https://sentinel.esa.int/web/sentinel/missions/sentinel2/overview)

One of Sentinel-2 objectives is to create a synergy with already existing land monitoring missions (e.g. USGS Landsat Thematic Mapper (TM) and Operational Land Imager (OLI) and the SPOT series). As visible in Figure 3. the bands of Sentine-2 MSI are comparable to the ones of the Landsat satellites.

The data products delivered to the public in a free and open access policy have 2 levels:

- Level-1A: Top-Of-Atmosphere reflectance distributed online;

- Level-2C: Bottom-Of-Atmosphere reflectance distributed online but can also be generated user side using the Sentinel-2 Toolbox (application part of the Sentinel Application Platform (SNAP));

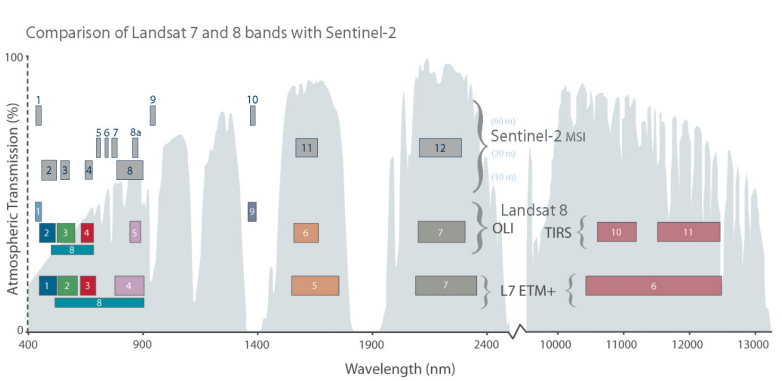

Figure 3. Comparison of Sentinel-2 with Landsat 7 and 8 (source: https://landsat.gsfc.nasa.gov/sentinel-2a-launches-ourcompliments-our-complements/ )

\subsection{Farm level monitoring: phenology assessment}

Timely and accurate information on crop growth and seasonal dynamics are increasingly needed to develop monitoring systems aimed to detect seasonal anomalies, support site specific management and estimate crop yield at the end of the season. In particular, frequent decametric information nowadays being provided exploiting the new generation of Earth Observation (EO) platforms are fundamental for farm level monitoring. The current availability of operational data from decametric multispectral systems, such as ESA Sentinel 2 and NASA/USGS Landsat-OLI, represents the fundamental precondition to develop solutions able to retrieve information at the agricultural management unity (i.e., the field). In this framework, during the summer school it was presented a study aimed at assessing the usefulness of dense time series of decametric Leaf Area Index (LAI) maps for phenological monitoring on rice-cultivated areas. More detail about this application can be found in (Boschetti et al. 2018). Decametric multi-source LAI time series were produced by the Department of Earth Physics and Thermodynamics (Universitat de València) by inverting the PROSAIL radiative transfer model with Gaussian process regression techniques (Campos-Taberner et al., 2016) using available Sentinel 2 and Landsat-7/8 mission's optical data acquired from early May up to the end of September for the year 2016. LAI maps at 10-30 m resolution, according to sensor source, were registered and resampled to Sentinel 2 images providing a 26 bands multi-temporal dataset for subsequent analysis. Phenological estimates were performed with a modified version of the PhenoRice algorithm (Boschetti et al., 2017). The time series of images were analyzed as follows: first, the LAI time series of each pixel was smoothed using a Savitzky-Golay two-iteration method able to assign weights to each LAI value according to EO product cloud mask information and LAI retrieval estimated accuracy. Subsequently, the smoothed signal, characterized by a 7 days regular LAI time series, was analyzed using PhenoRice to reconstruct estimate of the dates of occurrence of several rice crop phenological stages (crop establishment corresponding to 
DVS $=0$, emergence DVS $=1$, tillering DVS $=1.3$, flowering DVS $=2$, and maturity DVS $=4$ ) and to compute seasonal metrics (e.g., length of the vegetative and reproductive phases).

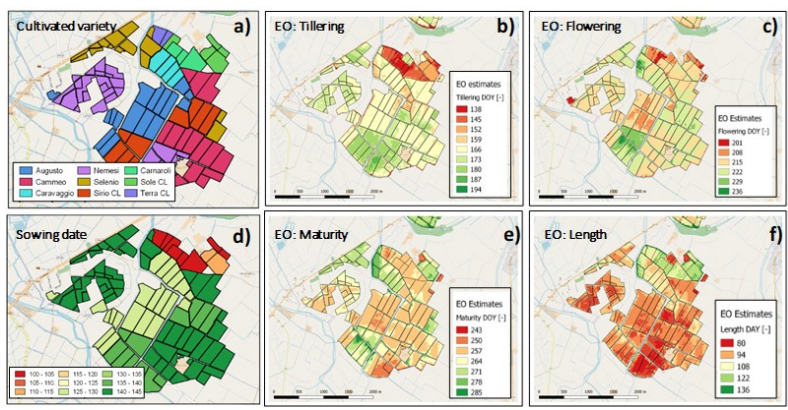

Figure 4. Examples of Phenological estimates at field level for a case study rice farm in Lomellina (North of Italy, Pavia province, latitude $45^{\circ} 15^{\prime} \mathrm{N} \pm 1.5^{\prime}$, longitude $8^{\circ} 34^{\prime} \mathrm{E} \pm 2^{\prime}, 112$ $\mathrm{m} \pm 15 \mathrm{~m}$ a.s.1.). Cultivated varieties (a) with different sowing dates (d) and phenological estimates @10 m resolution: tillering (b), flowering (c), maturity (e) and season length (f).

In figure 4. the variability of the crop condition in few kilometers according to cultivated varieties and agro-practices, in European context, is shown. It is therefore evident how parcel level information is needed to characterized and monitor such a complex condition. Estimates performances were assessed both exploiting field observation and modelling results (see Boschetti et al. 2018 for details). Differences between reference and EO estimates at flowering phenological stage (DVS 2) range between - 15 to 10 days, in the majority of the cases differences are comprised within 5 days and the RMSE is about 6.5 days. The preliminary test conducted in this study highlights how time series of decametric data can contribute to parcel-scale crop monitoring providing new insights on crop management practices, including information on cultivated varieties. This information can push towards crop modelling application for yield estimation at field scale and first test have been conducted to assess contribution in assimilation framework (Gilardelli et al., 2019).

\subsection{Crop monitoring: biophysical parameters, phenology and yield}

The analysis of high-spatial and temporal resolutions Sentinel 2 data is a powerful tool for crops monitoring. At the field level the estimation of crops' key biophysical parameters (Leaf Area Index - LAI, Leaf and Canopy Chlorophyll Density respectively $\mathrm{LCD}$ and $\mathrm{CCD}$ ) can be addressed by Empirical relationships with Vegetation Indexes (VIs) or by the inversion of Leaf and canopy radiative transfer (RT) models (PROSPECT+SAIL)

SNAP provides the opportunity to apply and compare both methods (i.e. empirical VIs' relationships and inversion of RT models) for the estimation of biophysical parameters from Sentinel 2 data. Many VIs sensitive to LAI, CCD and LCD can be obtained at 10 or $20 \mathrm{~m}$ resolution using SNAP band math or the SNAP VI processor, and used to apply crop-specific LAIVIs empirical relationships in band math. LAI, CCD and LCD estimates can be also obtained by using the SNAP Biophysical processor (Figure 4), employing a neural network (NNT) algorithm developed by INRA tailored for Sentinel-2 and trained using radiative transfer simulations from PROSPECT and SAIL RT models. Differences between empirical and RTM inversion-based estimates as implemented within SNAP for the two crops can be evaluated and critically discussed based on the capability to integrate prior crop-specific information, (e.g. average leaf inclination, taxonomy) critical for the accuracy of the estimation. While such capabilities are limited in the current release of the SNAP Biophysical processor, crop-specific empirical relationships of Biophysical parameters vs. VIs can be easily used in SNAP for empirical estimates of LAI, CCD and LCD.

Beside the temporal estimation of biophysical parameters, hightemporal resolution Sentinel 2 VIs Time-Series (TS) can be used for yield assessment and to extract key phenological metrics at the field level.

Phenological metrics that can be obtained include the onset of greenness (start of season), time of peak VI, maximum VI, rate of green-up, length of season, rate of senescence and timeintegrated area under the curve, a metric that can be used for yield forecast. Similarly, to the inversion of RT models current SNAP release incorporates basic tools for simple TS analysis.
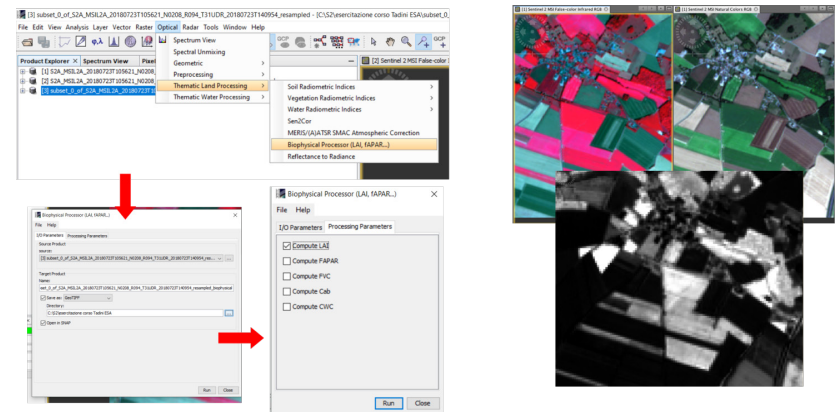

Figure 5. LAI estimate by using the SNAP Biophysical processor

Three multi-temporal Sentinel 2 part of images, corresponding to Piacenza province portions, geographically starting from 'Tadini Centre Locality Gariga di Podenzano Italy, towards south up to Emilian Apennines mountains are shown in Figure 5. The images, all acquired in 2018 (February, April, July), have been already processed and have been using for the Common Agricultural Policy Controls (CAP) in Italy; the band combination 8-11-4 (near infrared, short infrared, red) is the best, by literature and in operation, for agricultural monitoring and crop groups detection. Due to the different resolutions, short IR has $20 \mathrm{~m}$ pixel vs 10 of NIR, the CAP procedure suggests to do not apply the more correct pan sharpening method, which offers a better geometry (smoothing) but a worse spectral signature maintenance. This data set has been used for train the capability of the multi-temporal information of Sentinel 2 (72 possible yearly acquisitions in south Europe) to identify the crop phenological phases as additional 
information for the detection of 'cultivation groups' by spectral signatures.

\subsection{European CAP}

The Common Agriculture Policy (CAP) of European Union needs a verified money distribution to 8 million of EU farmers. Remote sensing geomatics applications allow to manage and control the single distributed subsidies overall the EU28 (27 next years) agricultural holdings.

Remote Sensing and the geomatics science have been accompanying and explaining the main purposes of this policy, the regulation evolution along the years, in parallel with the technology improvement, both in terms of resolution/spectral bands and satellite acquisition capacity. Particular focus is now assigned to Sentinel data new contribution, not only for improving the efficiency of the existing approach and methodology but, as often happens, for providing the base and the technical support of the new EU regulations: passing from "controls" at sample level to "monitoring" for the entire continental agro-environmental territory which will be applied in Europe after 2020. This different method, due to the Sentinel constellation availability, open to a continuous monitoring of each agronomic parcel, along the different phenological seasons in Europe. Obviously, this new systematic approach can be suggested and applied for other international applications/replications, even at regional level.

RPAS light devices (more diffused, especially for their major feasibility in authorization) are continuously growing in applications and in popular awareness, even for agriculture management and precision farming. However, at the important and satisfactory results (geometrical and thematic), must be always associated the corresponding limitations in terms of working time and costs (management and SW). Several official experiences have given an overview of the capability, through operational tests performed. For instance, the Italian Ministry of Agriculture asked and paid specific application trials aimed at evaluating the pasture graze ability, the soil erosion, the crop species distinguishing, the vineyard NDVI evolution, etc., with the goal to demonstrate when a benefit/cost balance can justify the light RPAS use. Generally, the above ratio appears positive when specific or complicate detections are requested, while for simple crop or land use analysis or updating the relative costs (devices, software and working time) seem too high for justifying the methodology against the traditional rapid field visits.

\subsection{Agriculture and Precision Farming}

Precision Farming and Forestry (PFF) are, nowadays, well sounding keywords for everyone involved in forest and agronomic practices. Nevertheless, at the state of the art, the same concept would be better addressed to as "Technological Forestry/Agriculture", being technology the most relevant issue that strongly characterizes this type of approach, making it different and, probably, "advanced", in respect of more traditional practices. Precision is something different: it concerns the quality of a measure (or of an intervention) and not exactly the way the problem is faced. It can be said that, ordinarily, people retain that technological approaches are more precise (and intelligent) than traditional one, generating this great misunderstanding.

To make "technological forestry/agriculture" a "precise" one, a great work has still to be done concerning the entire expected workflow: improvement of users' consciousness of both data and tools is the main challenge to ensure that PFF could be adopted at farm/forest company level. In particular, multispectral remote sensing from imaging systems is expected to heavily support this process. Recently, three facts have determined an exponential and sudden acceleration of remote sensing technology transfer to PFF: a) the availability of open access data archives of native and pre-processed global datasets (e.g NASA Landsat and MODIS, ESA Copernicus Sentinel data, etc.); b) the introduction into the market of low cost systems for image acquisition (i.e. RPAS, Remotely Piloted Aerial Systems) equipped with multispectral and thermal sensors; c) software development, that greatly has improved the degree of automation in data processing. Commercial players have been the firsts that approached the technology transfer, forecasting great income opportunities, especially in the agricultural context; this, generally, occurred with no care about the appropriate scientific consciousness; the high level of automation, given by software improvement, encouraged this trend. Surprisingly, traditional remote sensing experts initially did not participate to technology transfer. Consequently, this business-based approach is now showing some limitations mainly related to operators' unconsciousness about some technical aspects that are needed to make results and deductions reliable (Mondino, 2017). This is not a negligible issue, especially when quantitative measures are required to drive agronomic/forest interventions. The current moment is crucial: scientists have still not answered all the questions concerning properness and effectiveness of remote sensing to the operational compart (Borgogno-Mondino, 2017). Moreover, consistency of RS costs with those required by these at-lowincome sectors is not still demonstrated, nor the actual benefits that this technology can provide at farm/forest company level. Geomatics scientific community is therefore called to support this transitory phase by:

a) testing sensors and tools providing information concerning proper ranges of their usability;

b) transferring to users and sensors/sw producer's knowledge and skills about metric and spectral issues related to RS;

c) proposing and formalizing operational standards for both technology and data processing.

Among a great variety of unanswered questions, the following ones seem to require particular attention in this technology transfer phase:

a) how can automate data (free) processing to generate reliable spectral indices time series? Exploitation of all the available auxiliary information is expected to filter out bad observations at pixel level; 
b) which type of models can be adopt to translate spectral information into phenological/crop parameters? Can model be assumed as general or a continuous process of calibration has to be taken into account?

c) which is the minimum mapping unit to be considered in agriculture while monitoring crops from satellite? Have we to operate at pixel, cadastral parcel or segment level when reading the behavior of a cultivated field? The feeling is that the properness of spectral aggregation achieved at cadastral parcel level has to be overcome to get a true description of the ongoing phenomena.

d) How RPAS can effectively support agriculture and which is expected to be their relationship with satellite acquisitions?

\subsection{FLEX7Sentinel 3Tandem Mission}

Sentinel-3 is primarily an ocean mission, however, the mission is also able to provide atmospheric and land applications. The mission provides data continuity for the ERS, Envisat and SPOT satellites. Sentinel-3 makes use of multiple sensing instruments to accomplish its objectives; SLSTR (Sea and Land Surface Temperature Radiometer), OLCI (Ocean and Land Colour Instrument), SRAL (SAR Altimeter), DORIS, and MWR (Microwave Radiometer). An interesting future development of space observation is the combination between Sentinel 3 and FLEX in a tandem mission (Figure 6). The ESA's FLEX mission aims to provide global maps of vegetation fluorescence, which can be converted into an indicator of photosynthetic activity. These data would improve our understanding of how much carbon is stored in plants and their role in the carbon and water cycles. FLEX will be the first space mission designed to observe fluorescence by using a novel technique measuring the main part of the chlorophyll fluorescence spectrum that originates from the core of the photosynthetic machinery. Sun induced fluorescence will be measured with a high-resolution imaging spectrometer acquiring data in the 500-780 $\mathrm{nm}$ spectral range, with a sampling of $0.1 \mathrm{~nm}$ in the oxygen bands (759-769 nm and 686$697 \mathrm{~nm}$ ), while red edge, chlorophyll absorption and PRI (Photochemical Reflectance Index) will be computed at 0.5-2.0 nm. FLEX mission will orbit in tandem with Sentinel-3 of the Copernicus constellation. Taking advantage of Sentinel-3's optical and thermal sensors will lead to an integrated package of measurements to assess plant health. With the Sentinel-2 satellites also in orbit, there is a unique opportunity of using this data synergistically from all three missions for vegetation studies. Sentinel-3 instruments OLCI and SLSTR provide the needed atmospheric information, as well as contribute to retrieve vegetation information (LAI, chlorophyll) useful for the interpretation of the fluorescence signal.

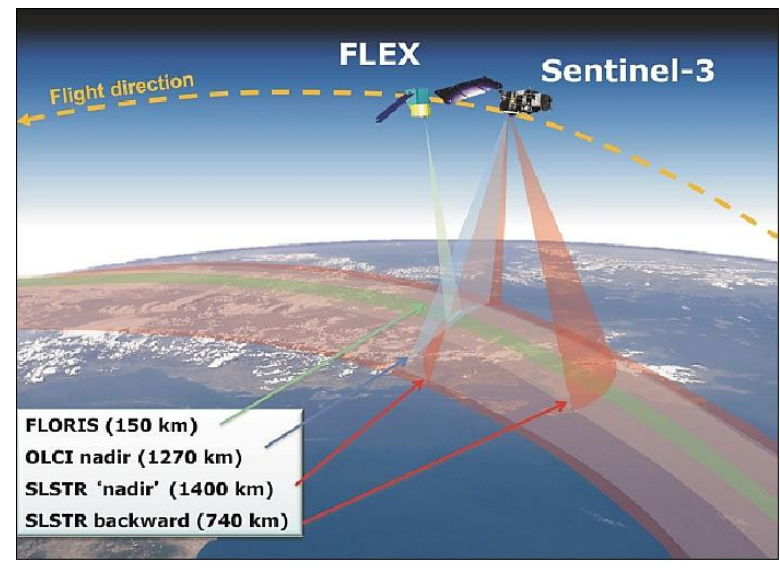

Figure 6. FLEX and Sentinel-3 joining forces (image credit: ESA)

\subsection{Drone survey and data elaboration}

This section is dedicated to illustrate the potentiality of drone survey as support tool for agriculture and precision farming. Nowadays UAV-RPAS-Drone instrument is a powerful a tool to produce high resolution orthophotos and high resolution Digital Elevation/Surface Models (DTMs and DSMs). An unmanned aerial vehicle (UAV), commonly known as drone or RPAS, is an aircraft without a human pilot aboard. UAVs are a component of an unmanned aircraft system (UAS); which include a UAV, a ground-based controller, and a system of communications between the two parts. Topographic modeling data processing is becoming more accessible and more widely used due to recent advances in digital photogrammetry methods and UAV-Drone instruments. Nowadays classic aerial photogrammetry survey is often replaced by Structure from Motion (SfM) techniques, where the non-metric camera is mounted on a UAV, and many photographs taken vertically or slantwise towards the ground. The SfM workflow generates, first of all, 3D models in arbitrary object coordinates and the precision of the model depends on image quality/quantity and on the $3 \mathrm{D}$ reconstruction algorithm. Moreover, the cartographic absolute accuracy of the final orthophoto and DSMs depends on the accuracy of the coordinates used in the georeferencing process. Commonly a complete drone survey includes a first step with area selection and drone system selection and setup, a second step that includes the flight plan definition and target positioning; the third step is the outside Drone flight including the GNSS target survey and final step is dedicated to data export and elaboration through Structure for Motion photogrammetric software (Agisoft Photoscan www.agisoft.com) in order to obtain Digital Surface Model and high resolution orthophoto of the surveyed area. 


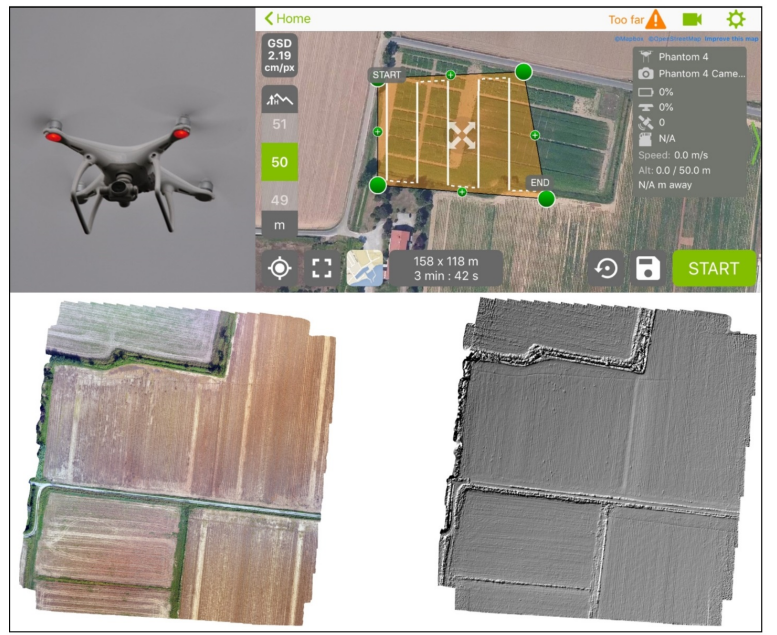

Figure 7. Elements of Drone Survey. From top left clockwise -

Drone Phantom 4 DJI, flight plan with parameters, DSM hillshade and orthophoto $2 \mathrm{~cm}$ GSD.

A synthetic process how to carry out the UAV-Drone survey is following:

- Define take-off, landing and flight sites, in particular for the survey with a UAV, always ensuring flight safety.

- Define the right flight planning together with drone pilot, ensuring adequate transverse and longitudinal overlaps to obtain good 3D intersections.

- Define position of well-distributed GCPs (Ground Control Points) by means of targets placed on the ground inside the survey area (and visible during the flights).

- Carry out differential, rapid static GNSS survey of all defined GCPs in order to extract 3D coordinates of the topographic network useful to georeferencing and scaling the 3D model and the derived cartographic products.

Once field surveys were carried out and all the data were collected, the main SfM data processing steps are:

- Matching photo survey with EXIF data;

- Building the point cloud in a local reference system;

- Texturing 3D model and building an orthomosaic;

- Georeferencing model in the cartographic reference system;

- Exporting the georeferenced orthomosaic and DSM;

During the survey setup and in the case of multispectral data comparison (i.e. with Sentinel images) the UAV-Drone can be equipped with multispectral and/or thermal sensors. The SfM techniques and software permits to use also multispectral or thermal images to produce final high resolution orthophoto. In this case we can use Drone orthophoto to analyze areas identified by Sentinel data in order to obtain a complete synoptic vision with infrared or thermal data.

\subsection{Sentinel Application Platform (SNAP) and Google Earth Engine}

A common architecture for all Sentinel Toolboxes has been developed and called the Sentinel Application Platform (SNAP) (Brockmann Consult, Array Systems Computing and C-S).

The SNAP architecture is ideal for Earth Observation processing and analysis due to several technological innovations: extensibility, portability, Modular Rich Client Platform, generic Earth Observation (EO) data abstraction, Tiled Memory Management, and a Graph Processing Framework. The image processing of Sentinel-2 data for agricultural applications is one of the key features available in SNAP. SNAP permits to build processing chains using its main features and to apply them to specific agricultural applications. In SNAP it is possible to open a Sentinel 2 image and to exploit the basic tools developed for image processing and analysis (e.g. raster tools, optical tools, band math and supervised classification). Basic computation of synthetic indexes (NDVI, EVI) is already available as default but can be modified in order to meet each user needs. Furthermore, it is possible to carry out supervised and unsupervised classifications. For example, random forest supervised classifications can be applied both on Level-1A and on Level-2C satellite images. SNAP is also suitable for land cover monitoring. For example, by combining 9 different Level-2C (one per month). it is possible to create a time-series stack. The result can be later on queried using the SNAP time series tool. This result is obtained by computing an NDVI for one image. Later, a chain graph is create using the simple NDVI. Afterwards, the generated graph it has been applied to all the 9 images using the batch processor. This permits to create the evolution of the NDVI for each pixel of the subset, as shown in the snapshot of the result (Figure 8). The obtained result can be later integrated or validated using higher resolution imagery e.g. aerial or RPAS.

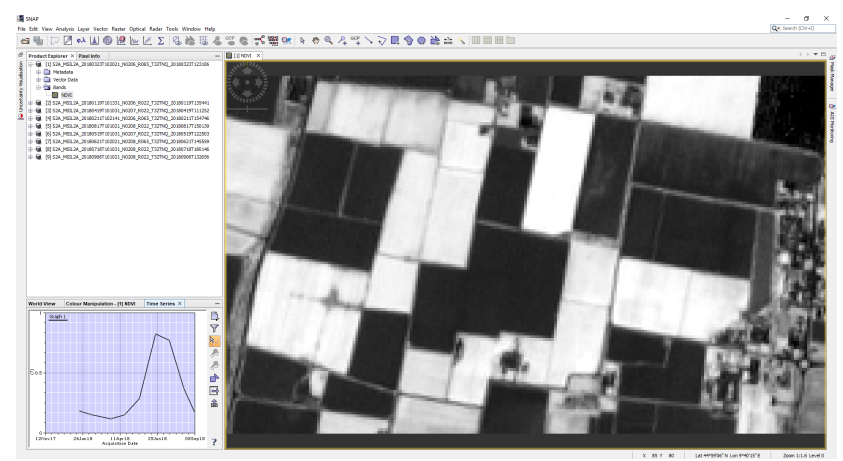

Figure 8. NDVI evolution using the Time series tool.

Google Earth Engine (EE) combines a multi-petabyte catalog of satellite imagery and geospatial datasets with planetary-scale analysis capabilities and makes it available for scientists, researchers, and developers to detect changes, map trends, and quantify differences on the Earth's surface. (https://earthengine.google.com/). The goal of the platform is to provide to its users the tools to perform analysis using earth 
observed data. Moreover, the platform is free to use, and every user owns the personal scripts and can decide whether to publish or keep them private. Fundamental is the comprehension of the main features, capabilities, and benefits for agricultural applications. These aspects are essential to give an overview of EE and in a second moment explore possible applications of the existing algorithms of the standard library of EE to remote sensed imagery.

Multi-temporal analysis can be implemented with ease in EE. For example, it is possible to perform a quick NDVI trend using the Sentinel-2 Level-1C imagery over a point of interest with only a few lines of code. In Figure 2 it is visible the interface of $\mathrm{EE}$, in the top-center the code used to filter one year the Sentinel-2 imagery over a specific point of interest and compute the NDVI, on the top-left the image the NDVI value of the point of interest (cyan) at the center of the image and the date of the value. This permits to have a quick overview of the situation of a certain crop. The main downside of EE is the unavailability of atmospherically corrected Sentinel-2 Level-2A data.

\section{CONCLUSIONS}

In this paper an overview of the Copernicus programme has been presented as a result of an organic collection and presentation of aspect related to the utilization and application of Sentinel data in agriculture applications. The field of applications is very wide and a basic approach has been introduced considering the fundamental aspect what could be taken in account when approaching with specific context.

Considering the relatively new possible approaches and the enormous set of automated data that are available and can be used, several unanswered questions are proposed stimulating the scientific community in approaching this frontier, opening strong operative possibilities to be studied and applied.

\section{REFERENCES}

Boschetti M., Busetto L., Ranghetti L., Garcia-Haro F.J., Campos-Taberner M. and Confalonieri R., 2018, "Testing Multi-Sensors Time Series of Lai Estimates to Monitor Rice Phenology: Preliminary Results," IGARSS 2018 - 2018 IEEE Int. Geoscience and Remote Sensing Symposium, Valencia, 2018, pp. 8221-8224. doi: 10.1109/IGARSS.2018.8518494

Busetto L., et al., 2017. Downstream services for rice crop monitoring in Europe: From regional to local scale. IEEE Journal of Selected Topics in Applied Earth Observations and Remote Sensing Volume: Issue 99. pp.1-19. 10.1109/JSTARS.2017.2679159

Borgogno Mondino, E., \& Gajetti, M. 2017. Preliminary considerations about costs and potential market of remote sensing from UAV in the Italian viticulture context. European Journal of Remote Sensing,50(1), 310-319.

Campos-Taberner M., García-Haro F.J., Camps-Valls G., GrauMuedra G., Nutini F., Crema A., Boschetti M., 2016. Multitemporal and multi resolution leaf area index retrieval for operational local rice crop monitoring. Remote Sensing of
Environment Volume 187, 15 December 2016, Pages 102-118 http://dx.doi.org/10.1016/j.rse.2016.10.009

Gilardelli C. , Stella T., Confalonieri R., Ranghetti L., CamposTaberner M., Garcia-Haro F.J, Mirco Boschetti M., (2019). Downscaling rice yield simulation at sub-field scale using remotely sensed LAI data. European Journal of Agronomy 103 (2019) 108-116. https://doi.org/10.1016/j.eja.2018.12.003

Gomarasca M.A., 2009. Basics of Geomatics. Springer, London New York, 656 pp ISBN: 9781402090134, DOI 10.1007/978-14020-9014-1

Nutini, F., Confalonieri, R., Crema, A., Movedi, E., Paleari, L., Stavrakoudis, D., Tabacchi, M., Cerioli, S., Tesio, F., Boschetti, M., 2018. Application of a satellite based approach to monitor rice nitrogen status and to support precision agriculture techniques, in: Società Italiana di Agronomia (Ed.), Atti Del XLVII Convegno Della Società Italiana Di Agronomia, Marsala (TP), 12-14 September 2018, pp. 17-19.

Mondino, E. B. 2017, June. Remote Sensing from RPAS in Agriculture: An Overview of Expectations and Unanswered Questions. In International Conference on Robotics in AlpeAdria Danube Region (pp. 483-492). Springer, Cham.

Oliver, C. J., Quegan, S. 1998. Understanding synthetic aperture radar images. Artech House, Inc., Norwood, MA.

Rossi L. Drone imagery use: pilot test in Italy - 22 MARS JRC Conference Lisbon (PT) 24-25 November 2017.

Rossi L. 2018. Controls with Remote Sensing vs Monitoring: the role of Sentinel2 - The actual use of imagery sessionWorkshop on checks and management of agricultural land in IACS - Vilnius Lithuania 28-30 May 2018.

Skriver, H., Mattia, F., Satalino, G., Balenzano, A., Pauwels, V. R. N., Verhoest, N. E. C., \& Davidson, M. 2011. Crop classification using short-revisit multi-temporal SAR data. IEEE Journal of Selected Topics in Applied EO and RS, 4(2), 423-431, DOI: 10.1109/JSTARS.2011.2106198 